\title{
A novel structure for cascade form adaptive notch filters
}

\author{
Soo-Chang Pei and Chien-Cheng Tseng \\ Department of Electrical Engineering, National Taiwan University, Taipei, Taiwan, ROC
}

Received 30 April 1992

Revised 28 September 1992

\begin{abstract}
The current cascade form adaptive notch filters have two different ways to adjust its parameters. First, each section is adapted individually (AI). Second, all sections are adapted simultaneously (AS). As to the complexity of tracking $p$ sinusoids, the AI structure is linear with $p$ but the AS structure is proportional to $p^{2}$. However, the AS structure has smaller bias in frequency estimates than that of the AI structure. In this paper, we will make a detailed comparison between the AI and AS structures by using a new prefilter technique. Then, we propose a novel structure which has the same complexity order as AIs and almost has the same lower frequency bias as ASs. Extensive computer simulations have been done to compare the performance of three structures under a wide range of tests.
\end{abstract}

Zusammenfassung. Für die übliche Kaskadenform für adaptive Kerbfilter gibt es zwei Möglichkeiten zur Einstellung der Parameter. Entweder wird jeder Block individuell adaptiert (AI) oder es erfolgt eine simultane Adaption aller Blöcke (AS). Entsprechend der Komplexität beim Nachführen von $p$ Sinussignalen ist die AI-Struktur linear in $p$ und die AS-Struktur proportional zu $p^{2}$. Andererseits weist die AS-Struktur einen geringeren Bias bezüglich der Frequenzschätzungen auf als die AI-Struktur. In dieser Arbeit wird ein detaillierter Vergleich zwischen der AI- und der AS-Struktur durchgeführt, wobei eine neue Vorfilter-Technik angewendet wird. Danach schlagen wir eine neue Struktur vor, die den gleichen Komplexitätsgrad wie die AI-Struktur aufweist, dabei aber den gleichen FrequenzBias besitzt wie die AS-Struktur. Zum Vergleich des Verhaltens der drei Strukturen werden ausführliche Computer-Simulationen wiedergegeben.

Résumé. Les filtres adaptatifs coupe-bande sélectifs en cascade actuels ajustent leurs paramètres de deux manières différentes. Dans la première, chaque section s'adapte individuellement (AI). Dans la seconde, toutes les sections s'adaptent simultanément (AS). En ce qui concerne la complexité de la poursuite de $p$ sinusoïdes, la structure AI est linéaire selon $p$ mais la structure AS est proportionnelle à $p^{2}$. Toutefois, la structure AS est caractérisée par un biais des estimées de fréquence plus petit que celui de la structure AI. Nous faisons dans cet article une comparaison détaillée entre les structures AI et AS à l'aide d'une technique nouvelle de pré-filtrage. Nous proposons ensuite une structure originale ayant le même ordre de complexité que la structure AI et presque le même biais en fréquence que la structure AS. Des simulations sur ordinateur approfondies ont été effectuées afin de comparer les performances des trois structures pour une gamme de tests étendue.

Keywords. Adaptive notch filter; cascade form structure.

\section{Introduction}

Recently, two major problems on processing multiple sinusoids in additive noise have received great attention. One is to estimate or track the sinusoidal frequencies $[4,15]$, the other is to enhance the sinu-

Correspondence to: S.-C. Pei, Department of Electrical Engineering, National Taiwan University, Taipei, Taiwan, ROC. soids from the background noise [16]. Several practical applications of these problems can be found in the area of communication, sonar, radar and biomedical signal processing.

The filter schemes which have been used to enhance and track noisy sinusoids are adaptive line enhancer (ALE) and adaptive notch filter (ANF). The ALE is generally realized by using Finite Impulse Response (FIR) filters, and the ANF is implemented as con- 
strained Infinite Impulse Response (IIR) filters. For this reason, the ANF have two main advantages over the ALE. First, the ANF is computationally efficient for the enhancement of sinusoids. Second, the ANF has a better model fitting than the ALE, since sinusoids in noise is an autoregressive moving average process. Therefore, the ANF is much more accurate in frequency estimates than the ALE.

In early work, most adaptive notch filters are implemented as direct form high order IIR filters [3, 8, 9, 11]. This form suffers from two disadvantages. One drawback is that stability monitoring is difficult, the other one is that the frequencies of the sinusoids need to be determined from the filter coefficients by using root finding or transfer function evaluation. Thus, many authors have used the cascade form adaptive notch filters to solve these two problems $[1,5,7,10]$.

In the recent approaches, the parameters of cascade form adaptive notch filters can be adjusted by two different ways. First, each section is adapted simultaneously (AS), as shown in Fig. 1(a) [5]. Second, all sections are adapted individually (AI), as depicted in Fig. 1(b) $[1,7,10]$. As to the complexity of tracking

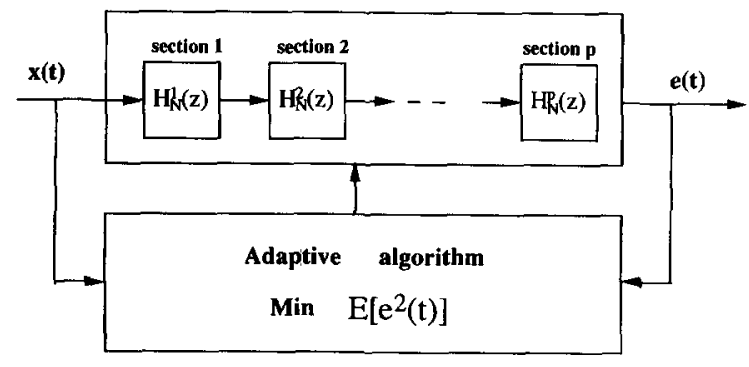

(a)

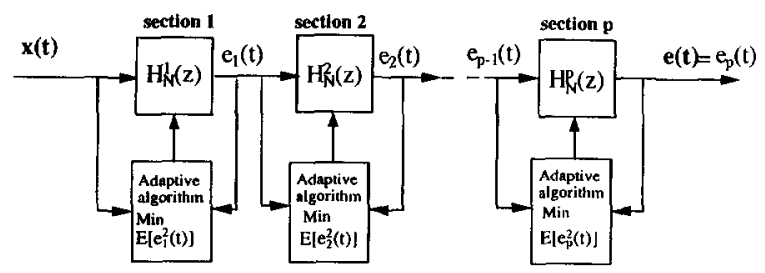

(b)

Fig. 1. (a) All sections are adapted simultaneously (AS). (b) Each section is adapted individually ( $\mathrm{AI}$ ), where $H_{\mathrm{N}}^{i}(z)$ denotes second order notch filter and $x(t)$ is multiple sinusoids embedded in additive noise. $p$ sinusoids, the AI structure is linear with $p$ but the AS structure is proportional to $p^{2}$. Thus, the AS structure is less efficient and more complicated than the AI structure in complexity. However, the AI structure minimizes the output mean square error of each section individually, this results in bias of frequency estimates even in the absence of noise. Thus the AS structure is much better than the AI structure in frequency estimation accuracy.

In this paper, we will make a detailed comparison between the AI and the AS structures by using a prefilter technique. From this point of view, it is obvious and easy to explain why the AS structure has smaller bias in frequency estimates than the AI structure. In addition, we propose a novel structure which has the same complexity order as AIs and almost has the same lower frequency bias as ASs.

The paper is organized as follows. First the problem statement is made in Section 2. Next the AS and AI structure is described in Section 3. The bias of frequency estimates in the AI and the AS structure will be analyzed in Section 4. Finally the novel structure is proposed in Section 5 and some computer simulations are presented.

\section{Problem statement}

Consider $p$ sinusoidal signals in a noise as follows:

$x(t)=\sum_{i=1}^{p} A_{i} \sin \left(\omega_{i} t+\phi_{i}\right)+v(t)$,

where $v(t)$ is a zero-mean white noise process with variance $\sigma^{2}$, and the phases $\left\{\phi_{i}\right\}$ are uniformly distributed on $[0,2 \pi)$. Besides, we assume that the sinusoids and noise are independent each other for all $t$. The signal-to-noise $\left(\mathrm{SNR}_{i}\right)$ is defined as $10 \log _{10} A_{i}^{2}$ / $\left(2 \sigma^{2}\right)$. Given the noisy samples $x(t)$, the problem is to estimate the sinusoidal frequency $\omega_{i}$ in real time by using cascade form adaptive notch filtering technique.

\section{The AS and AI structures}

In this section, we first describe the basic cell by which the AS and AI structures in Fig. 1 can be built 
and then demonstrate the difference between AS and AI in view of prefiltering.

\subsection{Basic cell}

The basic cell is shown in Fig. 2. It is comprised of the three following filters:

(a) The transfer function of the notch filter is

$H_{\mathrm{N}}^{i}(z)=\frac{1+a_{i} z^{-1}+z^{-2}}{1+\rho a_{i} z^{-1}+\rho^{2} z^{-2}}$,

where $\rho$ is the pole radius which is specified by the user. For stability, $\rho$ has to be smaller than one. In addition, the notch frequency $\omega_{\text {not }}^{i}$ and its $3 \mathrm{~dB}$ rejection bandwidth $\mathrm{BW}$ are given by

$\omega_{\text {not }}^{i}=\arccos \left(\frac{a_{i}}{-2}\right)$

and

$\mathrm{BW}=\pi(1-\rho)$.

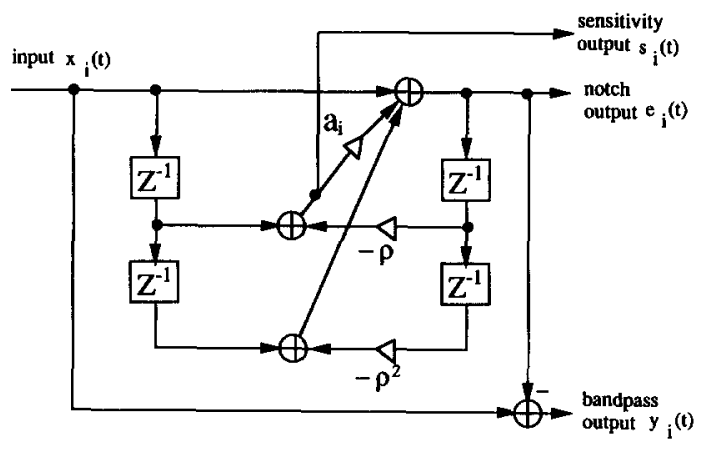

(a)

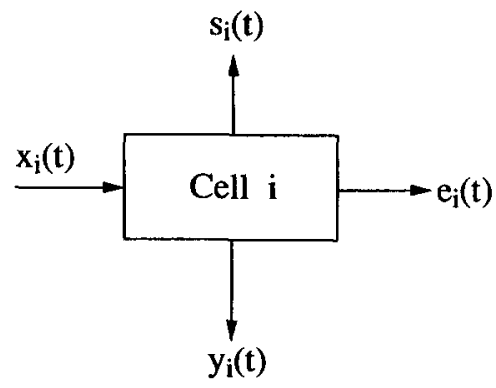

(b)

Fig. 2. (a) Signal flow graph of the basic cell. (b) Equivalent representation of the basic cell.
It is obvious that $H_{\mathrm{N}}^{i}\left(\omega_{\text {not }}^{i}\right)$ is equal to zero exactly.

(b) The bandpass filter is realized by subtracting the output of notch filter from its input. The transfer function is

$$
\begin{aligned}
H_{\mathrm{BP}}^{i}(z) & =1-H_{\mathrm{N}}^{i}(z) \\
& =\frac{(\rho-1) a_{i} z^{-1}+\left(\rho^{2}-1\right) z^{-2}}{1+\rho a_{i} z^{-1}+\rho^{2} z^{-2}} .
\end{aligned}
$$

Since $H_{\mathrm{N}}^{i}\left(\omega_{\text {not }}^{i}\right)=0$, then $H_{\mathrm{BP}}^{i}\left(\omega_{\text {not }}^{i}\right)=1$. This means that $H_{\mathrm{BP}}^{i}(z)$ has unit gain and zero phase at the notch frequency.

(c) The gradient or sensitivity filter can be obtained by the following method: From Fig. 2, we write the difference equation of the notch filter as

$$
\begin{aligned}
e_{i}(t)= & x_{i}(t)+a_{i} x_{i}(t-1)+x_{i}(t-2) \\
& -\rho a_{i} e_{i}(t-1)-\rho^{2} e_{i}(t-2) .
\end{aligned}
$$

By using a pseudolinear approximation $[9,14]$, we get the gradient component as

$\frac{\partial e_{i}(t)}{\partial a_{i}}=x_{i}(t-1)-\rho e_{i}(t-1)$.

Thus the gradient filter is given by

$H_{\mathrm{G}}^{i}(z)=\frac{(1-\rho) z^{-1}\left(1-\rho z^{-2}\right)}{1+\rho a_{i} z^{-1}+\rho^{2} z^{-2}}$.

In the following, we use the basic cell to construct the AS and the AI structures.

\subsection{The AS structure}

The basic configuration of the AS structure is shown in Fig. 1(a). It is clear that the transfer function from $x(t)$ to $e(t)$ is

$H(z)=\prod_{i=1}^{p} H_{\mathrm{N}}^{i}(z)$.

Let us define the gradient component as $g_{i}(t)=$ $\partial e(t) / \partial a_{i}$. Then using the sensitivity theory [2] and a pseudolinear assumption, we get the transfer function from $x(t)$ to $g_{i}(t)$ as follows:

$G_{i}(z)=H_{\mathrm{G}}^{i}(z) \prod_{k=1, k \neq i}^{p} H_{\mathrm{N}}^{k}(z)$ 
Combining (9) and (10), the overall AS structure is obtained in Fig. 3(a). From this structure, the notch output $e(t)$ and the gradient component $g_{i}(t)$ can be generated easily. Thus the filter parameters can be updated by

$a_{i}(t+1)=a_{i}(t)-\frac{\mu e(t) g_{i}(t)}{\left\|g_{i}(t)\right\|^{2}+p_{\min }}$.

The constant $p_{\min }$ is included to prevent division by zero when signals are not present [5]. From Fig. 3(a)

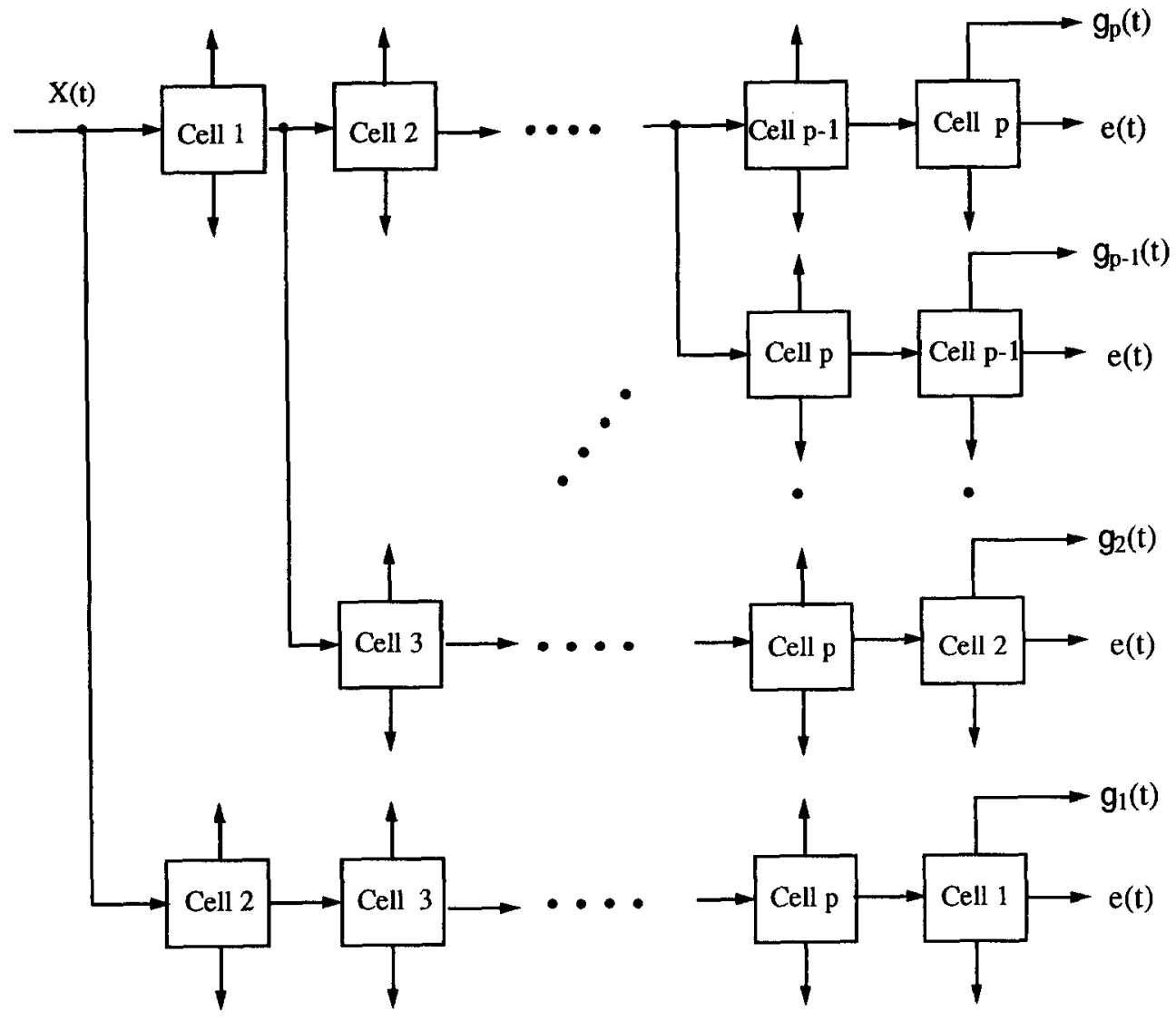

(a)

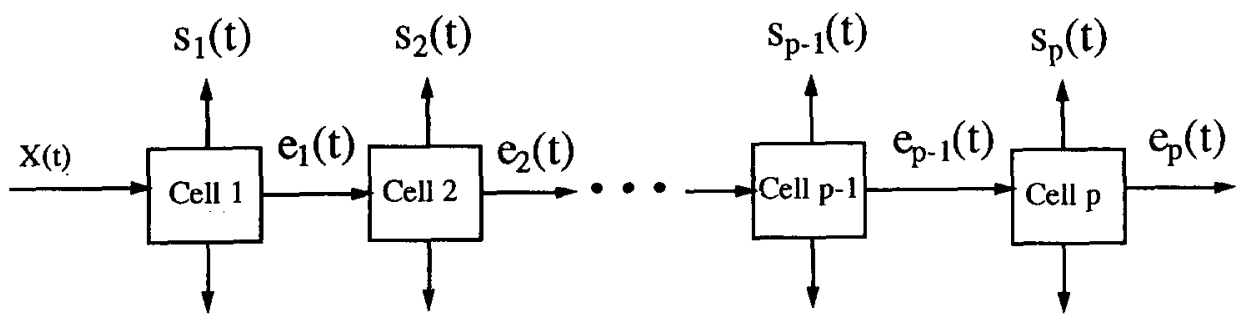

(b)

Fig. 3. (a) The AS structure for tracking $p$ sinusoids. (b) The AI structure for tracking $p$ sinusoids. 
it is easy to see the total number of the cells in the AS structure is $\frac{1}{2} p(p+1)+(p-1)$, so the complexity is proportional to $p^{2}$. Based on the AS structure, we summarize Algorithm 1 as follows.
Step 1. Use the AS structure in Fig. 3(a) to generate $e(t)$ and $g_{i}(t)$.

Step 2. Use (11) to update $a_{i}(t)$.

Step 3. Estimate frequency by $\hat{\omega}_{i}(t+1)$

$$
=\arccos \left(-\frac{1}{2} a_{i}(t+1)\right) \text {. }
$$

ALGORITHM 1. For each input sample $x(t)$

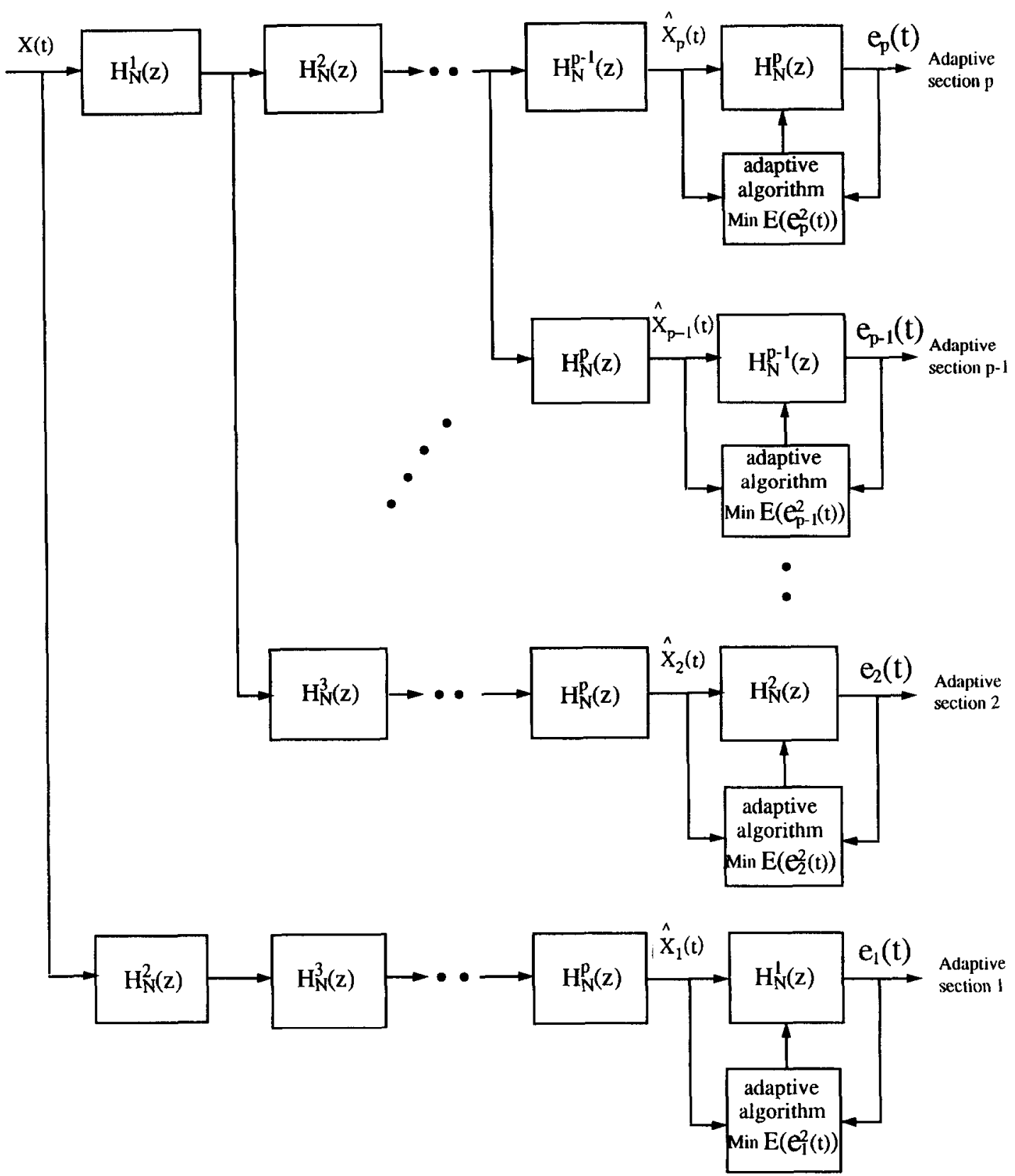

Fig. 4. A prefilter structure which is equivalent to the AS structure. 


\subsection{The AI structure}

The basic configuration of the AI structure is depicted in Fig. 1(b). Since each section minimizes its output mean square error, by using pseudolinear assumption the gradient component is given by $s_{i}(t)=\partial e_{i}(t) / \partial a_{i}$. Therefore, the final AI structure is shown in Fig. 3(b) and the filter parameters can be updated by

$a_{i}(t+1)=a_{i}(t)-\frac{\mu e_{i}(t) s_{i}(t)}{\left\|s_{i}(t)\right\|^{2}+p_{\min }}$.

It is obvious that the total number of cells in the AI structure is $p$, so its complexity is linear with $p$. Based on the AI structure,we summarize Algorithm 2 as follows:

ALGORITHM 2. For each input sample $x(t)$

Step 1. Use the AI structure in Fig. 3(b) to generate $e_{i}(t)$ and $s_{i}(t)$.

Step 2. Use (12) to update $a_{i}(t)$.

Step 3. Estimate frequency by $\hat{\omega}_{i}(t+1)$ $=\arccos \left(-\frac{1}{2} a_{i}(t+1)\right)$.

\subsection{Prefilter interpretation of the AS and AI structure}

It is worth to notice that the AS structure in Fig. 3(a) can be regarded as a prefilter structure shown in Fig. 4. This prefilter structure reveals that the procedure, which updates section $H_{\mathrm{N}}^{i}(z)$ in the AS structure, can be divided into the following two steps:

Step 1. Prefilter $x(t)$ by $\prod_{k=1, k \neq i}^{p} H_{\mathrm{N}}^{k}(z)$ to generate $\hat{x}_{i}(t)$, as shown in Fig. 4.

Step 2. $H_{\mathrm{N}}^{i}(z)$ adapts individually with input $\hat{x}_{i}(t)$.

In contrast, when $H_{\mathrm{N}}^{i}(z)$ is updated in the AI structure, its two steps are stated as follows:

Step 1. Prefilter $x(t)$ by $\prod_{k=1}^{i-1} H_{\mathrm{N}}^{k}(z)$ to generate $e_{i-1}(t)$, as shown in Fig. 1(b).

Step 2. $H_{\mathrm{N}}^{i}(z)$ adapts individually with input $e_{i-1}(t)$. Thus, the basic difference between the AS and the AI structure lies in the prefilter structure. In the next section, we will use this point of view to explain why the AS structure has a smaller bias of frequency estimates than the AI structure.

\section{Bias in AI and SI structure}

In the above, it can be seen that the AS structure requires more basic cells than the AI structure. Thus, we will show that the AS has a smaller frequency bias than the AI.

\subsection{Bias in AI structure}

Using the AI structure to track $p$ sinusoids in noise, the front cells in the structure have more than one sinusoids in its inputs. The performance surface analysis in $[1,10]$ have shown that each section only converges to one of $p$ sinusoids. The remaining sinusoids and noise will influence the adaption and cause frequency estimates bias. For understanding the fact, we derive the bias of the first section in the AI structure. The other sections can be derived by the similar ways. When Algorithm 2 converges, the stationary points of Section 1 are given by

$\mathrm{E}\left[s_{1}(t) e_{1}(t)\right]=0$.

Using the results in [6], (13) becomes

$F\left(a_{1}\right)=\sum_{i=1}^{p} \frac{A_{i}^{2}}{2} F_{i}\left(a_{1}\right)+\sigma^{2} F_{\mathrm{v}}\left(a_{1}\right)=0$,

where

$$
\begin{aligned}
& F_{i}\left(a_{1}\right) \\
& \quad=\frac{\left(a_{1}+2 \cos \left(\omega_{i}\right)\right)\left(1-\rho \cos \left(2 \omega_{i}\right)\right)}{\left[a_{1} \rho+\left(1+\rho^{2} \cos \left(\omega_{i}\right)\right)\right]^{2}+\left(1-\rho^{2}\right)^{2} \sin ^{2}\left(\omega_{i}\right)} \\
& F_{\mathrm{v}}\left(a_{1}\right)=\frac{a_{1}\left[1-\rho+\rho^{2}+\rho^{5}-2 \rho^{3}\left(\frac{1}{2} a_{1}^{2}-1\right)\right]}{(1+\rho)\left[1-2 \rho^{2}\left(\frac{1}{2} a_{1}^{2}-1\right)+\rho^{4}\right]} .
\end{aligned}
$$

Since the section 1 may converge to one of $p$ sinusoids, the solution to (14) in the vicinity of $-2 \cos \left(\omega_{i}\right)$, $i=1,2, \ldots, p$. Without loosing generality, let us assume that Algorithm 2 converges to the solution near

$\hat{a}_{1}=-2 \cos \left(\omega_{1}\right)$.

Then using Taylor's series expansion around $\hat{a}_{1},(14)$ becomes

$F\left(a_{1}\right) \approx F\left(\hat{a}_{1}\right)+F^{\prime}\left(\hat{a}_{1}\right)\left(a_{1}-\hat{a}_{1}\right)=0$,

where $F\left(\hat{a}_{1}\right)$ and $F^{\prime}\left(\hat{a}_{1}\right)$ may be evaluated from (14) easily. Thus the asymptotic bias of $a_{1}$ is 


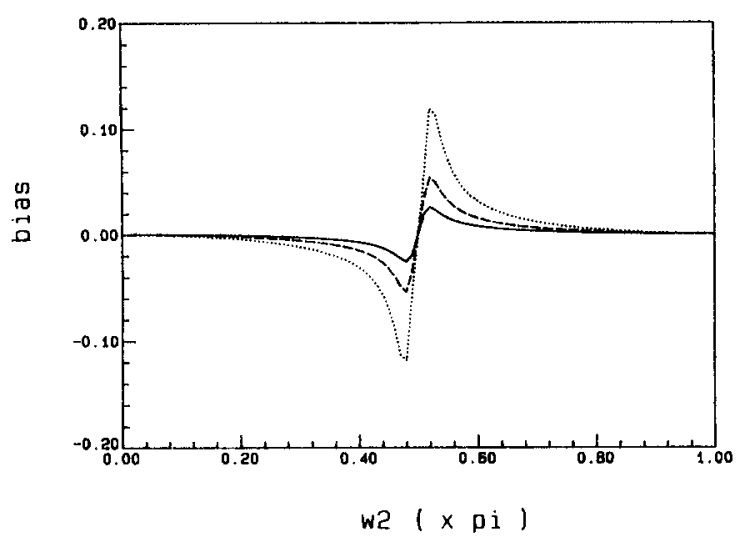

Fig. 5. Bias of $a_{1}$ in the first section of AI structure if $p=2$. Dotted line: $6 \mathrm{~dB}$; dashed line: $3 \mathrm{~dB}$; solid line: $0 \mathrm{~dB}$.

$\operatorname{bias}=a_{1}-\hat{a}_{1}=-\frac{F\left(\hat{a}_{1}\right)}{F^{\prime}\left(\hat{a}_{1}\right)}$.

A typical example of $p=2$ is shown in Fig. 5, which plots the bias of $a_{1}$ versus $\omega_{2}$ and $\mathrm{SNR}_{2}$ for $\omega_{1}=0.5 \pi$, $\mathrm{SNR}_{1}=3 \mathrm{~dB}$ and $\rho=0.95$. It is clear that the bias increases when the interfering sinusoid is strong or close to the sinusoid which section 1 converges to. Because the input of section $i$ is $x(t)$ prefiltered by $\prod_{k=1}^{i-1} H_{N}^{k}(z)$, the number of its interfering sinusoids is $p-i+1$. In particular, the input of the last section contains only one sinusoid in noise without any other interfering sinusoid. Thus, the last section will have the smallest frequency bias among all the sections.

\subsection{Bias in AS structure}

Since the AS structure can be viewed as a prefilter structure shown in Fig. 4, we will use this structure to explain why the AS have smaller bias than the AI. Beforehand, it is interesting to discuss the transient behavior of the structure in Fig. 4.

When each section in Fig. 4. has not converged at the beginning of adaption, the input signal $\hat{x}_{i}(t)$ of each adaptive section is almost the same as $x(t)$, because the frequency response of the notch filter has unit gain and zero group delay everywhere except at notch frequency. If some adaptive sections have converged, then their corresponding input sinusoids will be predeleted before entering the other nonconverged adaptive sections. This will make that nonconverged adaptive sec- tions only have the opportunity to converge to the remaining sinusoids.

After all adaptive sections have converged, the input signal of each adaptive section is only one sinusoid in almost undistorted white noise. Thus no interfering sinusoids remain to cause frequency estimate bias. Each adaptive section is just like the last section of the AI structure shown in Fig. 1(b). Since the last section in the AI structure have the smallest bias among all sections, the AS structure must have smaller bias than the AI structure.

\section{A novel structure}

Until now, we have explained that the AS structure has more complexity and less bias than the AI structure. Both are due to the major difference in the prefilter structure. In the section we will propose a novel prefilter structure in order that the complexity of AS can reduce to the same order as AI. This novel structure is mainly based on the following key property:

\section{KEY PROPERTY. Suppose}

$$
\begin{aligned}
& H_{\text {mul }}(z)=\prod_{i=1}^{q} H_{\mathrm{N}}^{i}(z) \\
& H_{\text {add }}(z)=\sum_{i=1}^{q} H_{\mathrm{N}}^{i}(z)-(q-1),
\end{aligned}
$$

then

$H_{\text {mul }}(z)=H_{\text {add }}(z)+\mathrm{O}\left((1-\rho)^{2}\right)$,

where $H_{\mathrm{N}}^{i}(z)$ is second order notch filter expressed in (2), and $\mathrm{O}(x)$ denotes a term which goes to zeros at least as $x$ when $x$ approaches zero [13].

PROOF. See Appendix A.

\section{REMARKS}

(a) From (4), the $3 \mathrm{~dB}$ rejection bandwidth $\mathrm{BW}$ of $H_{\mathrm{N}}^{i}(z)$ is $\pi(1-\rho)$. If the notch is very narrow, then $1-\rho$ approaches zero. Thus the key property becomes

$$
H_{\text {mul }}(z)=H_{\text {add }}(z)+\mathrm{O}\left(\mathrm{BW}^{2}\right) \text {. }
$$


Equation (19) means that we can use $H_{\text {add }}(z)$ to replace $H_{\text {mul }}(z)$ when $H_{\mathrm{N}}^{i}(z), i=1, \ldots, q$, have very narrow notch. A typical example of $q=2$ is illustrated as follows: Let

$H_{\mathrm{N}}^{1}(z)=\frac{1+0.5 z^{-1}+z^{-2}}{1+0.475 z^{-1}+0.9025 z^{-2}}$,

$H_{\mathrm{N}}^{2}(z)=\frac{1-0.5 z^{-1}+z^{-2}}{1-0.475 z^{-1}+0.9025 z^{-2}}$,

then

$H_{\mathrm{N}}^{1}(z) H_{\mathrm{N}}^{2}(z)=\frac{1+1.75 z^{-2}+z^{-4}}{1+1.5794 z^{-2}+0.8145 z^{-4}}$,

$H_{\mathrm{N}}^{1}(z)+H_{\mathrm{N}}^{2}(z)-1$

$$
=\frac{1+1.7506 z^{-2}+0.9905 z^{-4}}{1+1.5794 z^{-2}+0.8145 z^{-4}} .
$$

It is obvious that both transfer functions are almost the same, and their frequency responses look very similar as shown in Fig. 6.

(b) This key property has an intuitive explanation as follows:

Since

$$
\begin{aligned}
H_{\mathrm{add}}(z) & =\sum_{i=1}^{q} H_{\mathrm{N}}^{i}(z)-(q-1) \\
& =1-\sum_{i=1}^{q}\left(1-H_{\mathrm{N}}^{i}(z)\right),
\end{aligned}
$$

from (5), this expression becomes

$H_{\text {add }}(z)=1-\sum_{i=1}^{q} H_{\mathrm{BP}}^{i}(z)$

Note that $H_{\mathrm{BP}}^{i}(z)$ is a bandpass filter with unit gain and zero phase at the corresponding notch frequency of $H_{\mathrm{N}}^{i}(z)$. Moreover, the narrower notch $H_{\mathrm{N}}^{i}(z)$ has, the narrower passband $H_{\mathrm{BP}}^{i}(z)$ has. Therefore (20) implies that the function of $H_{\text {add }}(z)$ includes the following two parts: First, use $\sum_{i=1}^{q} H_{\mathrm{BP}}^{i}(z)$ to extract the sinusoidal signal from the input signal undistortedly. Second, subtract the extracted signal from the input

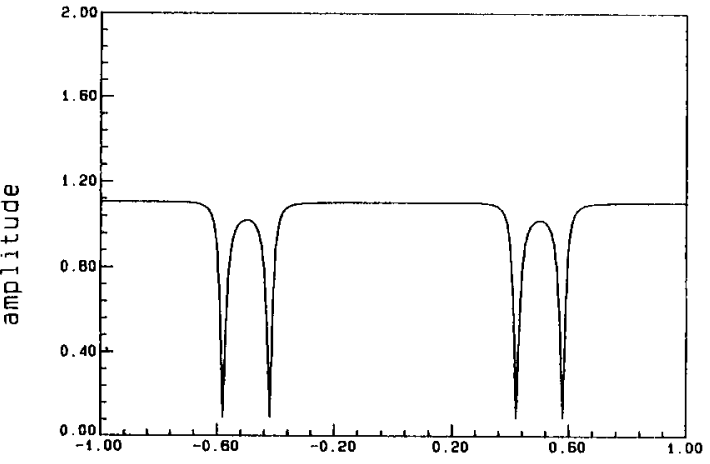

normalized frequency ( $x p i)$

(a)

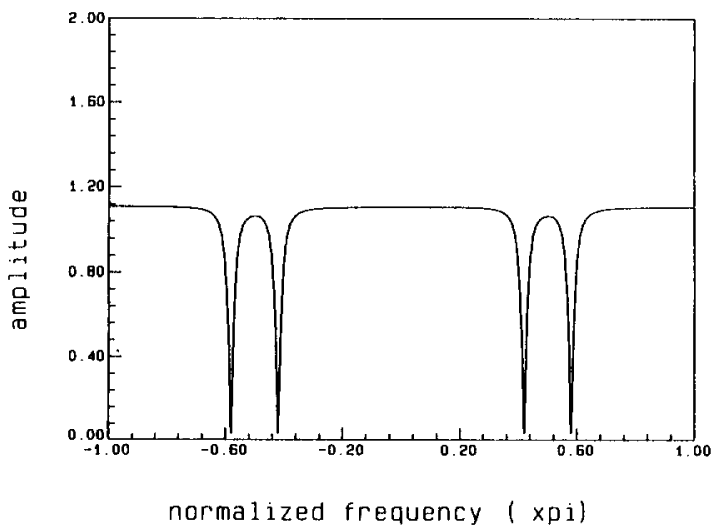

(b)

Fig. 6. Frequency response, (a) $H_{\mathrm{N}}^{1}(z) H_{\mathrm{N}}^{2}(z)$, (b) $H_{\mathrm{N}}^{1}(z)+$ $H_{\mathrm{N}}^{2}(z)-1$.

signal. The total effect of these two parts is equivalent to letting the input signal pass through $H_{\text {mul }}(z)$ with very narrow notch bandwidth.

(c) When $q=2$, from the derivation in Appendix A we have

$$
\begin{aligned}
H_{\text {mul }}(z)-H_{\text {add }}(z) & =\frac{(1-\rho)^{2} E_{1}(z) E_{2}(z)}{A_{1}(\rho z) A_{2}(\rho z)} \\
& =R(z) .
\end{aligned}
$$

We may choose $\rho$ such that

$$
\max _{0 \leqslant \omega \leqslant \pi}\left|R\left(\mathrm{e}^{\mathrm{j} \omega}\right)\right| \leqslant \varepsilon
$$

to guarantee that $H_{\text {add }}(z)$ is a good approximation to 


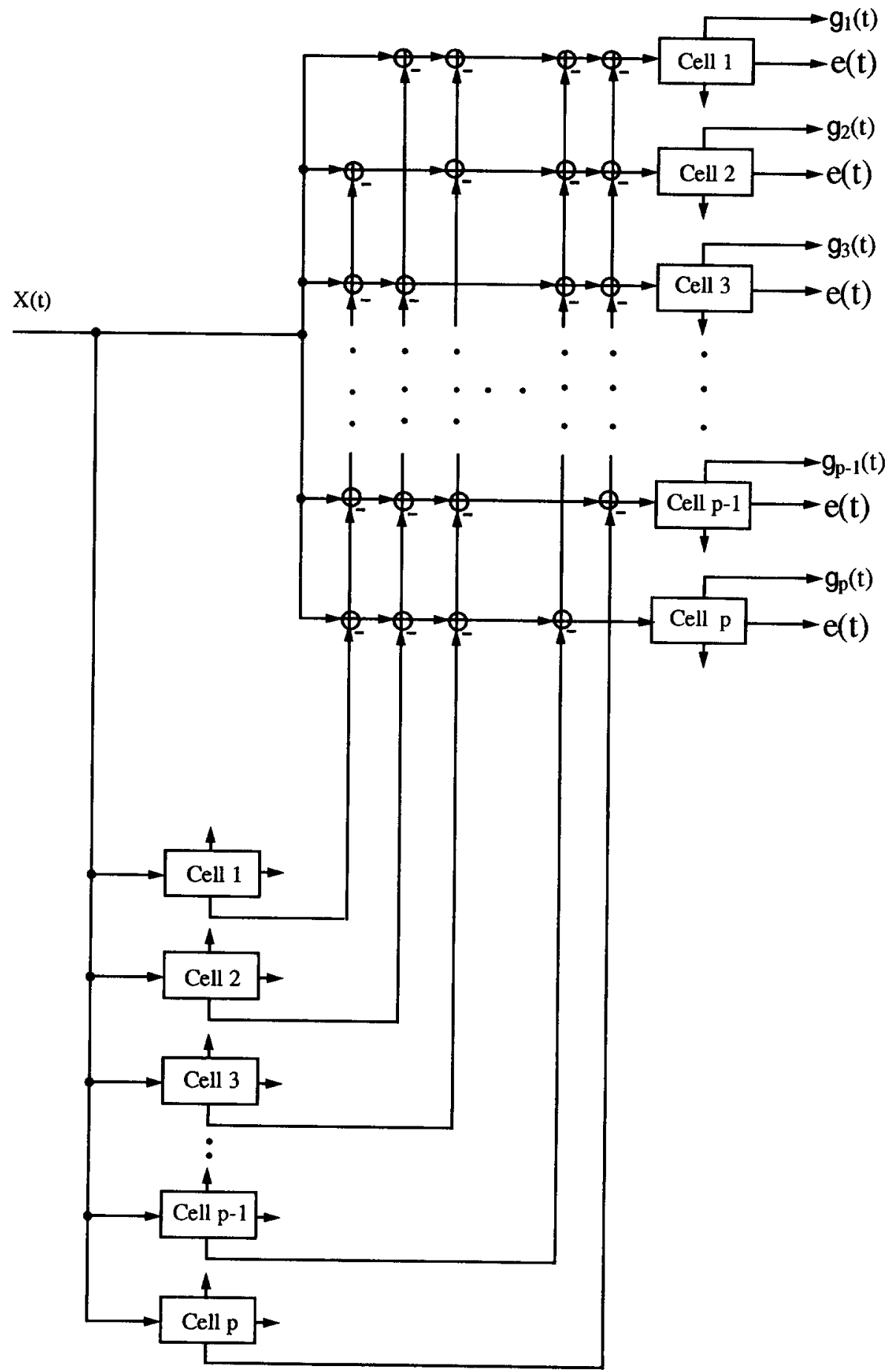

Fig. 7. A novel structure for tracking $p$ sinusoids. 
the $H_{\text {mul }}(z)$, where $\varepsilon$ is a small number. For example, if $a_{1}=-2 \cos (0.4 \pi), a_{2}=-2 \cos (0.5 \pi)$ and $\varepsilon=$ 0.07 , then $\rho$ must be greater than 0.98 such that (22) is valid.

Now, let us use this key property to reduce the complexity of the AS structure. According to (10), (19) and (20), the transfer function of gradient component in the AS structure can be written as

$G_{i}(z) \approx H_{\mathrm{G}}^{i}(z)\left(1-\sum_{k=1, k \neq i}^{p} H_{\mathrm{BP}}^{k}(z)\right)$

The narrower the notch bandwidth is, the better this approximation is. In our experience, when $\rho$ is greater than $0.95,(23)$ can be used very well. From (23) and Fig. 4, a novel structure can be constructed as shown in Fig. 7. The essence of this structure is the same as the AS structure, so the bias of frequency estimates will be very small. Besides, the number of cells is $2 p$. Thus its complexity is linear with $p$, i.e., the same as the AI structure. Based on this novel structure, we summarize Algorithm 3 as follows:

ALGORITHM 3. For each input sample $x(t)$

Step I. Use the novel structure in Fig. 7 to generate $e(t)$ and $g_{i}(t)$.

Step 2. Use (12) to update $a_{i}(t)$.

Step 3. Estimate the frequency by $\hat{\omega}_{i}(t+1)=$ $\arccos \left(-\frac{1}{2} a_{i}(t+1)\right)$.

\section{Experimental results}

Some computer simulations are performed with single precision floating-point on a VAX 3600 computer system to test the three algorithms in this paper. In all simulations, the notch frequencies are normalized with respect to the half sampling frequency.

EXAMPLE 1. Three input sinusoids with equal power. Referring to Fig. 8, this simulation compares the adaptive behavior of three algorithms. The input frequencies are at $0.6,0.7,0.8$, respectively. All sinusoids have equal power with an SNR of $3 \mathrm{~dB}$ and the notches are initialized at $0.2,0.3,0.4$, which are far from the input
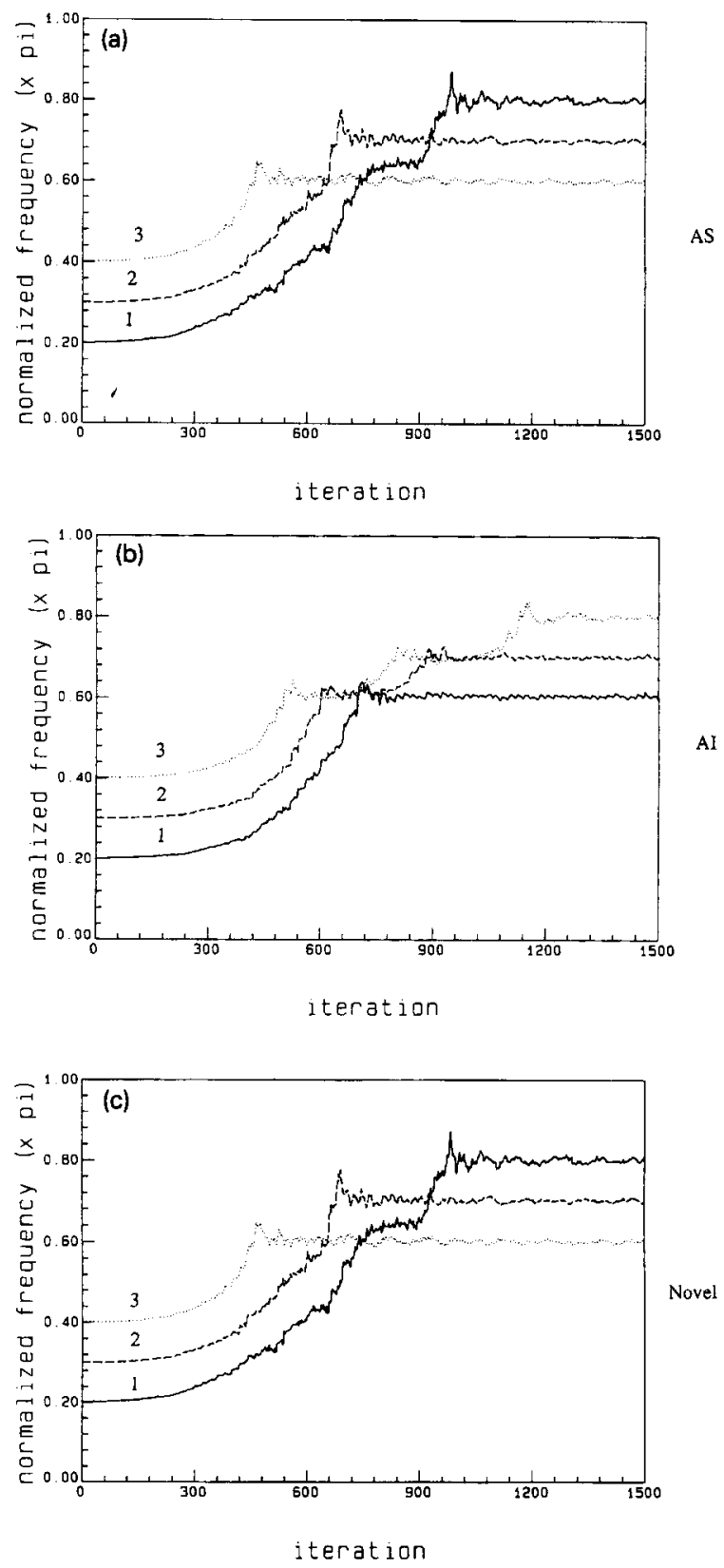

Fig. 8. Adaptive behavior of three algorithms. (a) Algorithm 1, (b) Algorithm 2, (c) Algorithm 3. Solid line: section 1; dashed line: section 2; dotted line: section 3 .

frequencies. The pole radius $\rho$ and the step size $\mu$ are fixed to be 0.95 and 0.005 during adaption. It can be seen that Algorithm 1 (AS structure) and algorithm 3 (Novel structure) have almost the same behavior as expected. In Algorithm 2 (AI structure), because each 
section is adapted individually, section 1 and section 2 converge to the same input sinusoid at the beginning of adaption. However, when section 1 have converged, corresponding sinusoid will be deleted before it inputs section 2 . Thus, section 2 changes its converged notch frequency from 0.6 to 0.7 . This means that the section 1 has higher 'priority' to choose a converged sinusoid than the remaining sections in the AI structure.

EXAMPLE 2. Two weak sinusoids in a strong sinusoid. Referring to Fig. 9, this example compares the adaptive behavior of three algorithms in a special case. That is, the input signal $x(t)$ consists of two weak sinusoids embedded in a very strong sinusoid. The input frequencies are at $0.2,0.4,0.6$, and their corresponding SNR are $20 \mathrm{~dB}, 0 \mathrm{~dB}, 0 \mathrm{~dB}$, respectively. The pole radius $\rho$ and the step size $\mu$ are fixed to be 0.95 and 0.005 and the initial notch frequencies are set at 0.333 , $0.5,0.667$, respectively. It is easy to see that the strong sinusoid has smaller 'ripple' and less 'influence' than the other two weak sinusoids in all three algorithms. However, the strong sinusoid causes larger bias of weak sinusoids' frequency in the $\mathrm{AI}$ structure than the other two structures.

EXAMPLE 3. Time varying pole radius $\rho$. In this example, we adapt the Nehorai's approach for stationary signal in which the pole radius is initialized to be a smaller value for fast convergence speed, but is increased exponentially according to

$\rho(t+1)=\rho_{0} \rho(t)+\left(1-\rho_{0}\right) \rho_{\infty}$

to improve the bias and signal enhancement [8]. In this simulation, $\rho(1)=0.7, \rho_{0}=0.99$ and $\rho_{\infty}=0.95$. The notch frequencies are initialized at $0.1,0.2,0.3$ and the frequencies of the input sinusoids are $0.5,0.6,0.7$. All sinusoids have an SNR of $3 \mathrm{~dB}$ and the step size $\mu$ is chosen as 0.005 . Referring to Fig. 10, it can be seen that all three algorithms converge to the correct frequencies. Note that Algorithm 3 does not suffer undesired problems even though the initial $\rho$ is chosen as 0.7. However, in order to guarantee that the novel structure is a good approximation to the AS structure, the $\rho$ (1) had better not be too far from unity at the beginning of the adaptive process.
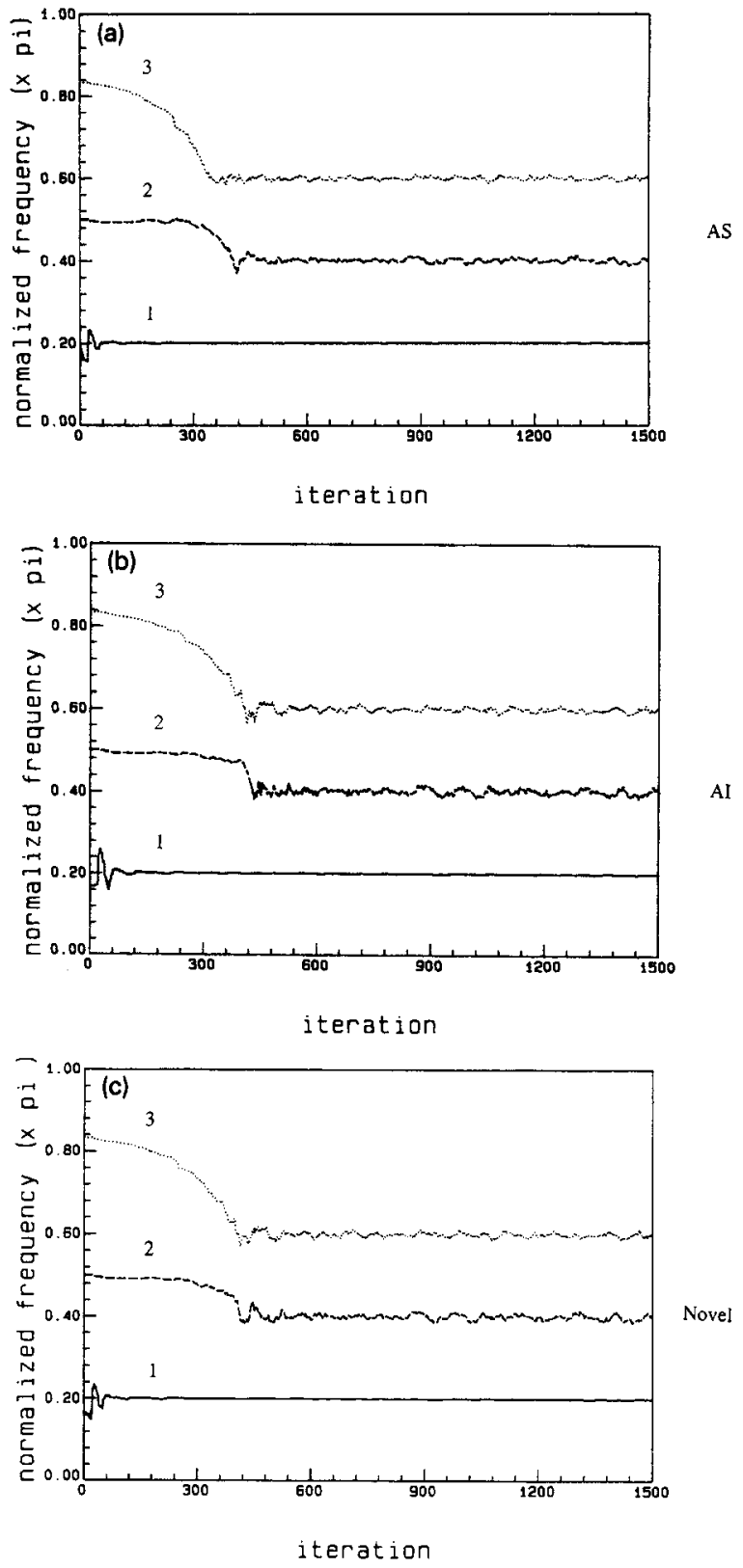

Fig. 9. Adaptive behavior for two $0 \mathrm{~dB}$ sinusoids in a $20 \mathrm{~dB}$ sinusoid. (a) Algorithm 1, (b) Algorithm 2, (c) Algorithm 3. Solid line: section 1; dashed line: section 2; dotted line: section 3 .

EXAMPLE 4. Tracking the time-varying sinusoids. In this example, we concentrate on the use of three algorithms for tracking the nonstationary signals, wherein the frequency of input sinusoids is assumed to be time 


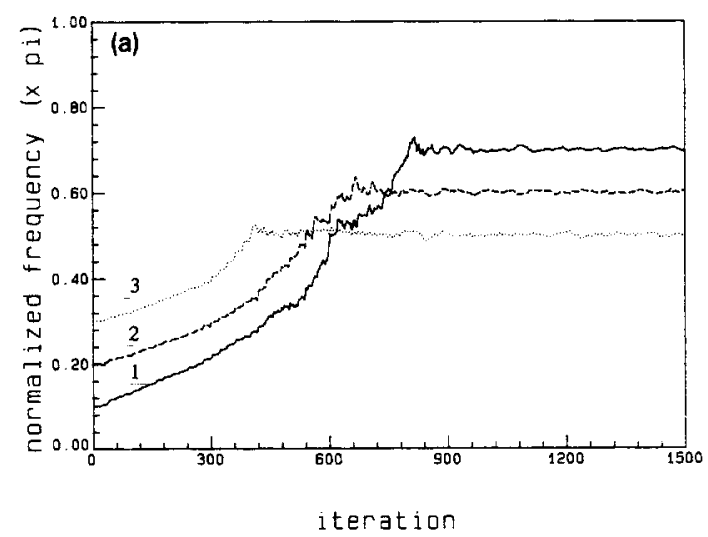

AS

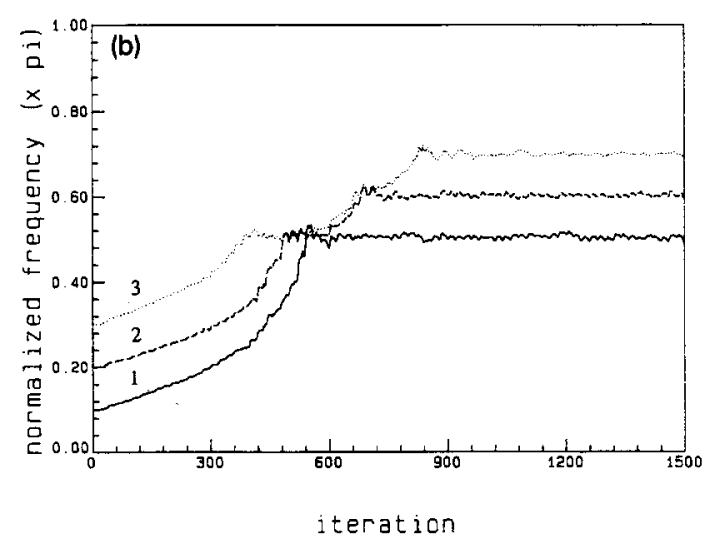

AI
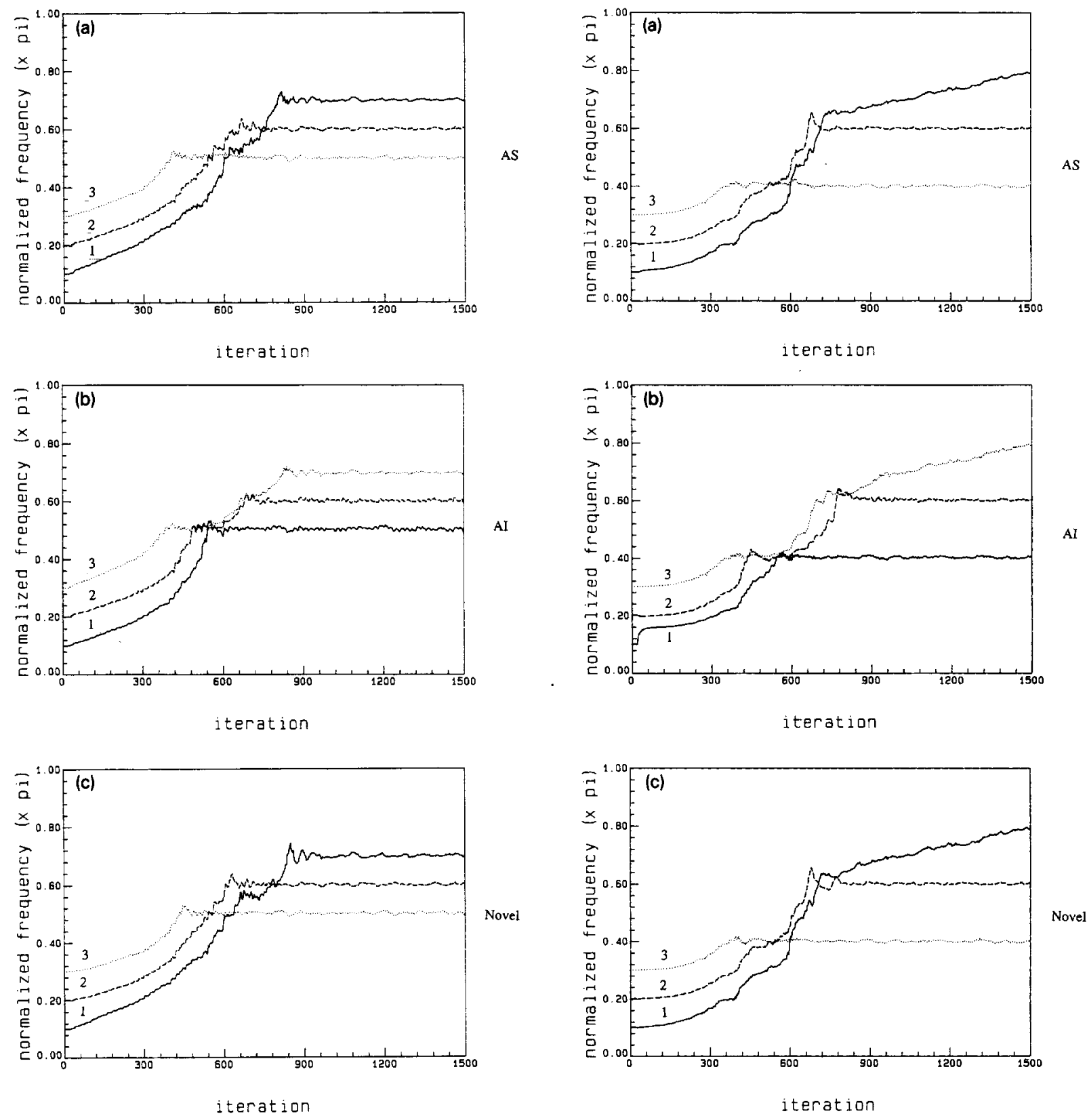

Fig. 10. Adaptive behavior for time varying pole radius. (a) Algorithm 1, (b) Algorithm 2, (c) Algorithm 3. Solid line: section 1; dashed line: section 2; dotted line: section 3 .

Fig. 11. Tracking behavior of the linear chirp signal. (a) Algorithm 1, (b) Algorithm 2, (c) Algorithm 3. Solid line: section 1; dashed line: section 2; dotted line: section 3 .

varying. Three typical cases are investigated below [12]. In each case, the pole radius $\rho$ and the step size $\mu$ are fixed at 0.95 and 0.005 .

(a) Tracking chirp signal.

First, we consider the tracking of a linear chirp signal.

The input signal $x(t)$ consists of two sinusoids with fixed frequencies at $0.4,0.6$ and a frequency increasing chirp signal of the following form:

$\sqrt{2} \sin \left(0.5 \pi t+0.001 \pi t^{2}\right)$.

Signal Processing 


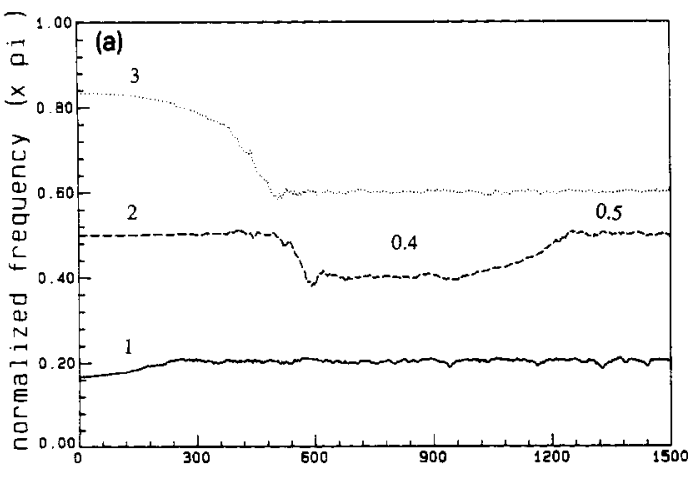

iteration

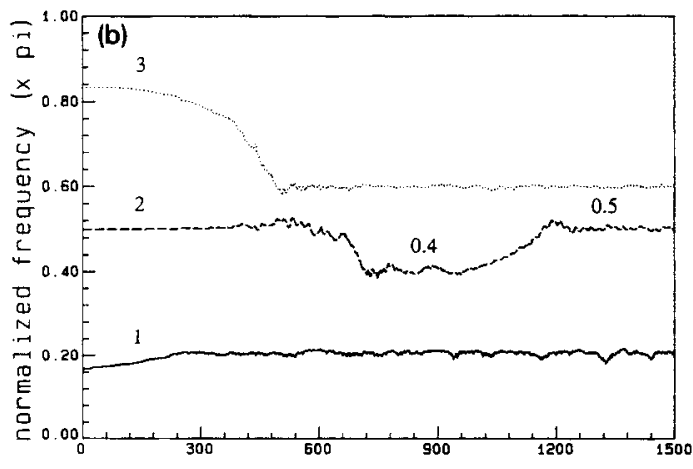

iteration

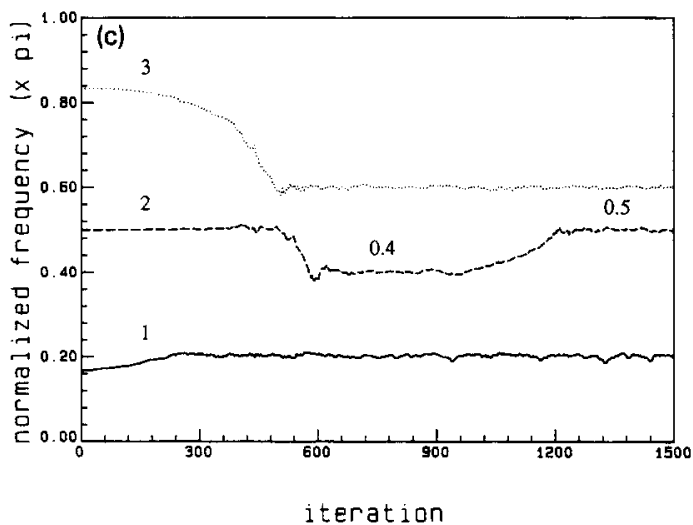

Fig. 12. Tracking behavior of the sinusoid with step-changed frequency. (a) Algorithm 1, (b) Algorithm 2, (c) Algorithm 3. Solid line: section 1; dashed line: section 2; dotted line: section 3 .

All signal components have an SNR of $3 \mathrm{~dB}$ and the notch frequencies are initialized at $0.1,0.2$ and 0.3 . Figure 11 clearly indicates that the tracking capabilities of the three algorithms are good.

(b) Tracking the sinusoid with step changed frequency.

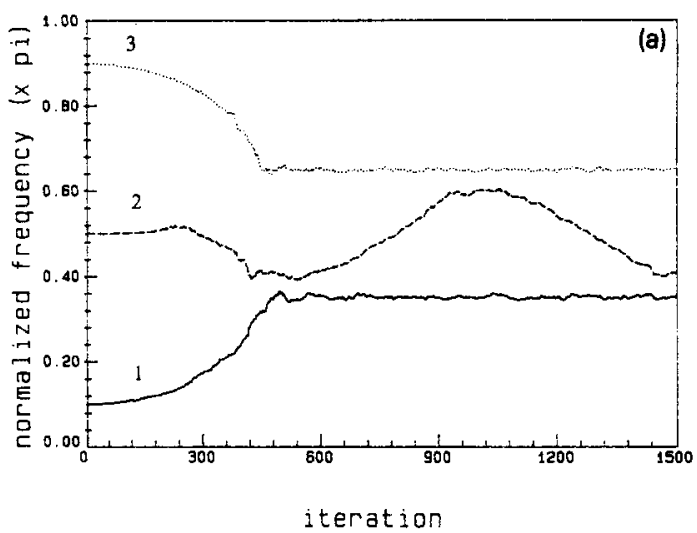

AS
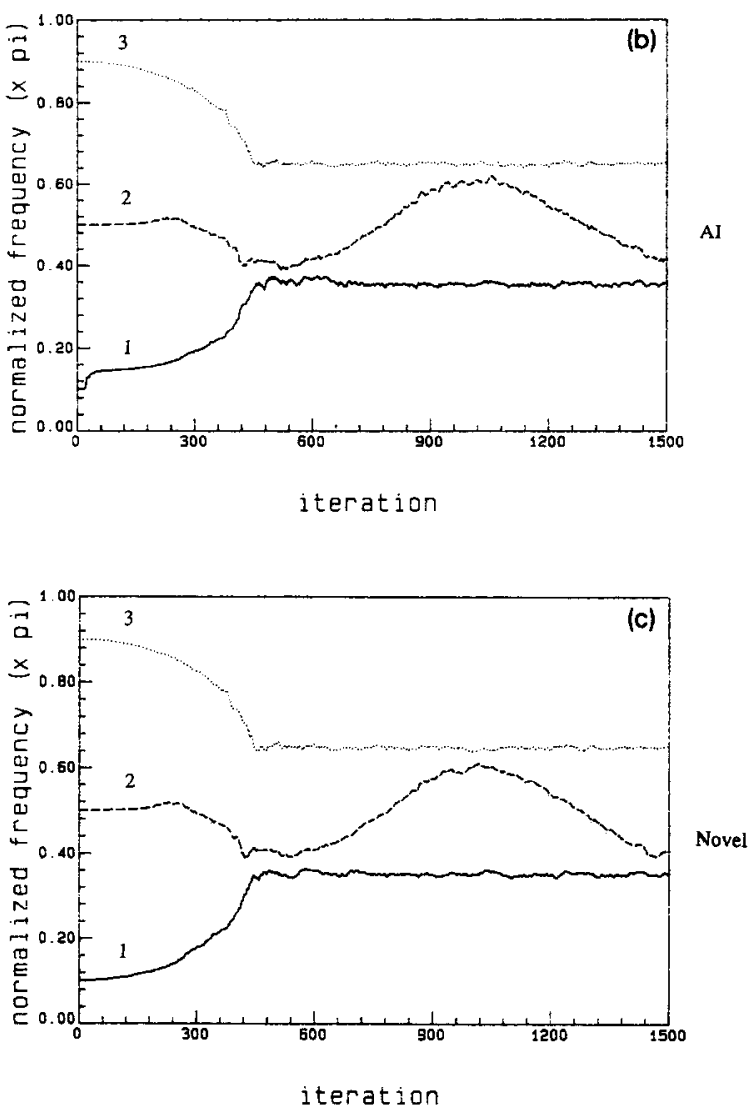

Fig. 13. Tracking behavior of the sinusoid with its frequency varied sinusoidally. (a) Algorithm 1, (b) Algorithm 2, (c) Algorithm 3. Solid line: section 1; dashed line: section 2; dotted line: section 3 .

Next, we consider the input signal, in which the sinusoidal frequencies are initially at $0.2,0.4$ and 0.6 , respectively for the first 1000 sample points, after which they are suddenly step changed to $0.2,0.5$ and 0.6. All sinusoids have an SNR of $3 \mathrm{~dB}$ and the notches 
Table 1

Statistical results of Algorithm 1

\begin{tabular}{|c|c|c|c|c|c|c|c|}
\hline \multirow[b]{2}{*}{$N$} & \multirow{2}{*}{$\begin{array}{l}\text { SNR } \\
(\mathrm{dB})\end{array}$} & \multicolumn{3}{|c|}{ Bias $\times 10^{-5}$} & \multicolumn{3}{|c|}{ Standard deviation $\times 10^{-5}$} \\
\hline & & $\hat{f}_{1}$ & $\hat{f}_{2}$ & $\hat{f}_{3}$ & $\hat{f}_{1}$ & $\hat{f}_{2}$ & $\hat{f}_{3}$ \\
\hline \multirow[t]{3}{*}{1000} & 1 & 21.07 & 15.96 & -46.74 & 69.92 & 44.87 & 61.77 \\
\hline & 5 & 5.832 & 6.217 & -21.25 & 43.24 & 26.09 & 38.85 \\
\hline & 9 & 1.931 & 4.527 & -8.893 & 27.43 & 16.54 & 24.70 \\
\hline \multirow[t]{4}{*}{1500} & 1 & 33.02 & 30.10 & -41.19 & 64.70 & 53.99 & 64.59 \\
\hline & 5 & 7.361 & 14.02 & -17.67 & 39.93 & 32.23 & 39.09 \\
\hline & 9 & 1.633 & 6.738 & -8.690 & 24.76 & 19.76 & 24.21 \\
\hline & 13 & -0.4619 & 3.248 & -5.299 & 15.42 & 12.28 & 15.14 \\
\hline
\end{tabular}

Table 2

Statistical results of Algorithm 2

\begin{tabular}{|c|c|c|c|c|c|c|c|}
\hline \multirow[b]{2}{*}{$N$} & \multirow{2}{*}{$\begin{array}{l}\text { SNR } \\
(\mathrm{dB})\end{array}$} & \multicolumn{3}{|c|}{ Bias $\times 10^{-5}$} & \multicolumn{3}{|c|}{ Standard deviation $\times 10^{-5}$} \\
\hline & & $\hat{f}_{\mathrm{I}}$ & $\hat{f}_{2}$ & $\hat{f}_{3}$ & $\hat{f}_{1}$ & $\hat{f}_{2}$ & $\hat{f}_{3}$ \\
\hline \multirow[t]{3}{*}{1000} & 1 & 422.9 & 45.92 & -29.17 & 121.6 & 56.82 & 70.85 \\
\hline & 5 & 375.0 & 31.97 & -8.982 & 72.02 & 32.60 & 41.78 \\
\hline & 9 & 354.7 & 26.57 & -2.265 & 44.21 & 19.87 & 25.43 \\
\hline \multirow[t]{4}{*}{1500} & 1 & 391.1 & 57.88 & -27.61 & 103.7 & 39.27 & 51.87 \\
\hline & 5 & 356.6 & 37.76 & -9.841 & 61.39 & 23.23 & 31.93 \\
\hline & 9 & 343.8 & 29.41 & -3.332 & 37.69 & 14.47 & 19.95 \\
\hline & 13 & 339.1 & 25.86 & -1.097 & 23.52 & 9.108 & 12.54 \\
\hline
\end{tabular}

Table 3

Statistical results of Algorithm 3

\begin{tabular}{|c|c|c|c|c|c|c|c|}
\hline \multirow[b]{2}{*}{$N$} & \multirow{2}{*}{$\begin{array}{l}\text { SNR } \\
\text { (dB) }\end{array}$} & \multicolumn{3}{|c|}{ Bias $\times 10^{-5}$} & \multicolumn{3}{|c|}{ Standard deviation $\times 10^{-5}$} \\
\hline & & $\hat{f}_{1}$ & $\hat{f}_{2}$ & $\hat{f}_{3}$ & $\hat{f}_{1}$ & $\hat{f}_{2}$ & $\hat{f}_{3}$ \\
\hline \multirow[t]{5}{*}{1000} & 1 & 30.86 & 19.71 & -35.23 & 69.74 & 54.04 & 56.70 \\
\hline & 5 & 10.93 & 5.922 & -13.85 & 41.82 & 31.19 & 35.23 \\
\hline & 9 & 3.597 & 0.8851 & -6.229 & 25.65 & 18.92 & 22.08 \\
\hline & 13 & 0.6616 & -0.7689 & -3.725 & 15.91 & 11.69 & 13.88 \\
\hline & 17 & -0.5483 & -1.186 & -3.046 & 9.993 & 7.293 & 8.741 \\
\hline \multirow[t]{5}{*}{1500} & 1 & 13.59 & 23.00 & -28.59 & 51.87 & 57.11 & 50.15 \\
\hline & 5 & 1.237 & 8.124 & -1.232 & 33.20 & 34.35 & 30.66 \\
\hline & 9 & -2.447 & 2.411 & -6.402 & 21.23 & 21.00 & 19.27 \\
\hline & 13 & -3.162 & 0.2593 & -4.291 & 15.53 & 12.99 & 12.21 \\
\hline & 17 & -2.947 & -0.5186 & -3.594 & 8.599 & 8.092 & 7.739 \\
\hline
\end{tabular}


are initialized at $0.333,0.5$ and 0.667 . It can be seen from Fig. 12 that the three algorithms track this step change very well.

(c) Tracking the sinusoid with its frequency varied sinusoidally.

Finally, we study the tracking of the input signal which consists of two sinusoids with fixed frequencies at 0.35 , 0.65 and a sinusoid with its frequency varied sinusoidally:

$\sqrt{2} \sin (0.5 \pi t+50 \sin (0.002 \pi t))$.

The SNRs of all sinusoids are $3 \mathrm{~dB}$ and the notch frequencies are initialized at $0.1,0.5$ and 0.9 . Figure 13 shows that the frequency tracking errors are very small in all three algorithms.

EXAMPLE 5. Statistical performance of three algorithms. In this example, we demonstrate the statistical behavior of three algorithms. The input frequencies are at $0.45,0.5$ and 0.55 . All sinusoids have an equal power with different SNRs and the notches are initialized at $0.333,0.5,0.667$. The pole radius $\rho$ and the step size $\mu$ are fixed at 0.95 and 0.005 during adaption. Tables 1 to 3 summarize the bias and standard deviation of the notch frequencies calculated from 40 independent trials. Algorithms 1 and 3 have almost the same statistical results as expected. Also, we see that the smallest bias $\hat{f}_{3}$ in Algorithm 2 is almost the same as the bias of each $\hat{f}_{i}$ in Algorithms 1 and 3 . This result is consistent with our previous prediction.

\section{Conclusion}

A novel structure for cascade from adaptive notch filters has been developed in this paper. It can reduce the complexity greatly from order $p^{2}$ to order $p$, and it also has the same lower frequency bias as ASs. Extensive computer simulations have been done to test the performance of this new structure.

\section{Appendix A. Proof of key property}

Let us define

$$
\begin{aligned}
& A_{i}(z)=1+a_{i} z^{-1}+z^{-2}, \\
& A_{i}(\rho z)=1+\rho a_{z}^{-1}+\rho^{2} z^{-2} . \\
& E_{i}(z)=-a_{i} z^{-2}-(1+\rho) z^{-2},
\end{aligned}
$$

then

$$
A_{i}(\rho z)=A_{i}(z)+(1-\rho) E_{i}(z) .
$$

Moreover,

$H_{\mathrm{mul}}(z)=\prod_{i=1}^{q} \frac{A_{i}(z)}{A_{i}(\rho z)}=\frac{\prod_{i=1}^{q} A_{i}(z)}{\prod_{i=1}^{q} A_{i}(\rho z)}$

and

$$
\begin{aligned}
H_{\mathrm{add}}(z)= & \sum_{i=1}^{q} \frac{A_{i}(z)}{A_{i}(\rho z)}-(q-1) \\
= & \left\{\sum_{j=1}^{q}\left[A_{j}(z) \prod_{i=1, i \neq j}^{q} A_{i}(\rho z)\right]\right. \\
& \left.-(q-1) \prod_{i=1}^{q} A_{i}(\rho z)\right\} / \prod_{i=1}^{q} A_{i}(\rho z) .
\end{aligned}
$$

So,

$H_{\text {mul }}(z)-H_{\text {add }}(z)=\frac{Q(z)}{\prod_{i=1}^{q} A_{i}(\rho z)}$,

where

$$
\begin{aligned}
Q(z)= & \prod_{i=1}^{q} A_{i}(z)-\sum_{j=1}^{q}\left[A_{j}(z) \prod_{i=1, i \neq j}^{q} A_{i}(\rho z)\right] \\
& +(q-1) \prod_{i=1}^{q} A_{i}(\rho z) .
\end{aligned}
$$

Making use of (27),

$$
\begin{aligned}
Q(z)= & \prod_{i=1}^{q} A_{i}(z) \\
& -\sum_{j=1}^{q}\left\{A_{j}(z) \prod_{i=1, i \neq j}^{q}\left[A_{i}(z)+(1-\rho) E_{i}(z)\right]\right\} \\
& +(q-1) \prod_{i=1}^{q}\left[A_{i}(z)+(1-\rho) E_{i}(z)\right] .
\end{aligned}
$$

Note that the sum of the first term and the third term in (29) is 


$$
\begin{aligned}
\prod_{i=1}^{q} A_{i}(z)+(q-1) \prod_{i=1}^{q}\left[A_{i}(z)+(1-\rho) E_{i}(z)\right] \\
=q \prod_{i=1}^{q} A_{i}(z) \\
\quad+(q-1) \sum_{j=1}^{q}\left[A_{j}(z) \prod_{i=1, i \neq j}^{q} E_{i}(z)\right](1-\rho) \\
\quad+\mathrm{O}\left((1-\rho)^{2}\right) .
\end{aligned}
$$

Moreover, by expanding and combining, the second term in (29) becomes

$$
\begin{aligned}
\sum_{i=1}^{q} & \left\{A_{j}(z) \prod_{i=1, i \neq j}^{q}\left[A_{i}(z)+(1-\rho) E_{i}(z)\right]\right\} \\
=q \prod_{i=1}^{q} A_{i}(z) & \\
& +(q-1) \sum_{j=1}^{q}\left[A_{j}(z) \prod_{i=1, i \neq j}^{q} E_{i}(z)\right](1-\rho) \\
& +\mathrm{O}\left((1-\rho)^{2}\right) .
\end{aligned}
$$

Substituting (30) and (31) into (29), we get

$Q(z)=\mathrm{O}\left((1-\rho)^{2}\right)$.

From (28) and (32), we obtain

$$
H_{\mathrm{mul}}(z)=H_{\mathrm{add}}(z)+\mathrm{O}\left((1-\rho)^{2}\right)
$$

and the proof is completed.

\section{References}

[1] J.F. Chicharo and T.S. Ng, "Gradient-based adaptive IIR notch filtering for frequency estimation", IEEE Trans. Acoust. Speech Signal Process., Vol. ASSP-38, May 1990, pp. 769777 .
[2] U.Forssen, "Simple derivation of adaptive algorithms for arbitrary filter structure', Electron. Letters, Vol. 26, October 1990, pp. 1764-1766.

[3] B. Friedlander and J.O. Smith, "Analysis and performance evaluations of an adaptive notch filter", IEEE Trans. Inform. Theory, Vol. IT-30, March 1984, pp. 280-295.

[4] S.M. Kay, Modern Spectral Estimation, Theory \& Application, Prentice Hall, Englewood Cliffs, NJ, 1988.

[5] T. Kwan and K. Martin, "Adaptive detection and enhancement of multiple sinusoids using a cascade IIR filter", IEEE Trans. Circuits and Systems, Vol. CAS-36, July 1989, pp. 937-947.

[6] P.L. Mack and L. Pearlstein. "Asymptotic accuracy of an adaptive notch filter with pseudolinear regression approximation', Proc. Conf. Information Sciences and Systems, Baltimore, MD, USA, 1989, pp. 92-97.

[7] N.K. M'Sirdi, H.A. Tjokronegoro and I.D. Landau, “An RML algorithm for retrieval of sinusoids with cascade notch filter", Internat. Conf. Acoust. Speech Signal Process., New York, USA, April 1988, pp. 2484-2487.

[8] A. Nehorai, "A minimal parameter adaptive notch filter with constrained poles and zeros", IEEE Trans. Acoust. Speech Signal Process., Vol. ASSP-33, August 1985, pp. 983-996.

[9] T.S. Ng, "Some aspects of an adaptive digital notch filter with constrained poles and zeros', IEEE Trans. Acoust. Speech Signal Process., Vol. ASSP-35, February 1987, pp. 158-161.

[10] M.R. Petraglia, S.K. Mitra and J. Szczupak, "Adaptive sinusoid detection using IIR notch filters and multirate techniques", Internat. Conf. Circuits and Systems, New Orleans, LA, USA, May 1990, pp. 271-274.

[11] D.V.B. Rao and S.Y. Kung, "Adaptive notch filtering for the retrieval of sinusoids in noise" , IEEE Trans. Acoust. Speech Signal Process., Vol. ASSP-32, August 1984, pp. 791-802.

[12] B.D. Rao and R. Peng, "Tracking characteristics of the constrained IIR adaptive notch filter', IEEE Trans. Acoust. Speech Signal Process., Vol. ASSP-36, September 1988, pp. 1466-1479.

[13] P. Stoica and A. Nehorai, "Performance analysis of an adaptive notch filter with constrained poles and zeros"' IEEE Trans. Acoust. Speech Signal Process., Vol. ASSP-36, June 1988, pp. 911-919.

[14] P. Stoica and A. Nehorai, "Convergence analysis of an adaptive pseudolinear-regression notch filtering algorithm", Circuits Systems Signal Process., Vol. 10, No. 2, 1991, pp. 245-259.

[15] P. Stoica, R.L. Moses, B. Friedlander and T. Söderström, "Maximum likelihood estimation of the parameters of multiple sinusoids from noisy measurements", IEEE Trans. Acoust. Speech Signal Process., Vol. ASSP-37, March 1989, pp. 378392.

[16] B. Widrow and S.D. Stearns, Adaptive Signal Processing, Prentice Hall, Englewood Cliffs, NJ, 1985. 